\title{
The response of essential tremor to propranolol: evaluation of clinical variables governing its efficacy on prolonged administration
}

\author{
S CALZETTI, ${ }^{*}$ LJ FINDLEY,* E PERUCCA, $\dagger$ A RICHENS $\ddagger$
}

From the Medical Research Council, Neuro-Otology Unit, Institute of Neurology, ${ }^{*}$ National Hospital, Queen Square, London, UK, Istituto di Farmacologia Medica, Universita di Pavia, Pavia, Italy, $\dagger$ and the Department of Pharmacology and Therapeutics, Welsh National School of Medicine, Cardiff, Wales $\ddagger$

SUMMARY The factors influencing the response of essential tremor to prolonged administration of propranolol (120 mg daily for two weeks followed by $240 \mathrm{mg}$ daily for a further two weeks) were investigated in a double-blind, cross-over, placebo-controlled study in 16 patients. Hand tremor was assessed by means of accelerometers with off-line computer analysis. Propranolol was found to be superior to placebo only at the higher dosage regimen (240 mg daily). At this dosage, the median reduction in tremor amplitude (as compared to the control value) was by $45 \%$. The response to the drug (expressed as percentage change in tremor amplitude) was correlated positively with the control amplitude $\left(r_{s}=0.71, p<0.01\right)$ and negatively (but more weakly) with the control peak frequency of tremor $\left(r_{s}=-0.53, p<0.05\right)$. In the patients with hand tremor greater than $6 \times 10^{-3} \mathrm{~cm}$ hand displacement the tremor amplitude was reduced by $65 \%$, as compared to only $17 \%$ in patients whose tremor amplitude was below this limit. No statistically significant relationship could be found between percentage change in tremor amplitude and duration of the disorder, age of the patients, degree of cardiac $\beta$-blockade or serum propranolol levels. The results suggest that patients with small tremor amplitude should not be treated with propranolol unless their tremor becomes severely aggravated under conditions of excessive adrenergic discharge.

The $\beta$-adrenoceptor antagonist propranolol is the drug of first choice in the symptomatic control of essential tremor but not all the patients respond ${ }^{1-3}$ and a wide range of individual responses are reported. ${ }^{4-6}$ In the past attempts have been made to identify factors potentially useful in predicting the therapeutic outcome. Dupont et $a^{1}{ }^{1}$ and Murray? found that the effect of chronic propranolol treatment was better in younger patients and in those with shorter duration of tremor. This contrasts with the observations of Teravainen $e t a^{2}$ and Larsen ${ }^{8}$ who found propranolol treatment more effective in older patients and in those with lower frequency of tremor. In our previous study, ${ }^{9}$ patients with larger amplitude and lower frequency of tremor showed a better response to a single oral dose of propranolol

Address for reprint requests: Dr Stefano Calzetti, Istituto di Clinica Neurologica, Via del Quartiere, 443100 Parma (Italy).

Received 12 November 1982

Accepted 9 December 1982
$(120 \mathrm{mg})$ than those with smaller amplitude and higher frequency. The present investigation was designed to verify whether the same factors are of value in predicting the therapeutic efficacy of propranolol following chronic administration.

\section{Methods}

Patients Sixteen patients with mild to severely disabling essential tremor ( 8 male and 8 female) aged between 20 and 72 years (mean age 43 years) and attending the outpatient clinics at the National Hospital for Nervous Diseases, Queen Square, gave their informed consent to participate in the study. The diagnosis was established on the basis of the clinical history and detailed general and neurological examination accompanied by ancillary laboratory investigations where necessary. All patients had been symptomatic for at least one year prior to the study. In nine patients there was a family history of tremor affecting the hands and/or the head. Fifteen patients had previously taken part in a single oral dose study with propranolol.9 None of the patients was receiving any drug therapy for tremor at the time of the study but nine of them 
had been previously treated with propranolol (dose range 80-240 mg daily). Patients with a history of excessive alcohol consumption, congestive cardiac failure, heart block, diabetes mellitus and asthma were excluded.

Protocol The study was crossover, randomised, doubleblind and placebo-controlled. In addition to propranolol and placebo, each patient was also given a course of metoprolol, $150 \mathrm{mg}$ daily for two weeks followed by 300 $\mathrm{mg}$ daily for two weeks. (Details on the comparative efficacy of metoprolol and propranolol are reported elsewhere $\left.^{10}\right)$. Each treatment period lasted for 4 weeks. Propranolol was given at two different dosage regimens (120 $\mathrm{mg}$ daily for two weeks followed by $240 \mathrm{mg}$ daily for the remaining two weeks). The active drug or placebo were given in three divided daily doses. Patients were instructed to take the previous dose about two hours before testing. Tremor assessment was carried out at the end of each dosage period (that is, every two weeks) by using piezoresistive linear accelerometers (Endevco 7265-10) taped to the dorsum of the hands, with their sensitive axes orientated in the vertical plane, in the $2 \mathrm{nd}$ interspace $1 \mathrm{~cm}$ proximal to the metacarpophalangeal joints. These devices weighed $6 \mathrm{~g}$ and had a frequency response extending from the steady state acceleration to $300 \mathrm{~Hz}$ with a sensitivity of $50 \mathrm{mV} / \mathrm{g}$ ( $g=$ acceleration of gravity). Tremor was recorded with the patient seated, the forearms supported up to the wrist and the hands unsupported maintained outstretched horizontally in a pronated posture. On each recording session three separate tremor recordings each of approximately one minute duration were obtained at 5 minute intervals. To minimise the effects of fatigue the hands were allowed to rest freely between the individual recordings within each session. The patient's position was monitored throughout the recordings by closed circuit video. The derived accelerometric signals were amplified and recorded simultaneously on paper using an electrostatic recorder (Elema-Shonander) and on magnetic tape (HewlettPackard 3968 A) for off-line tremor analysis. Measurements of pulse rate were obtained after a ten minute period of rest in the supine position and repeated after 1,2 and 3 minutes of standing (the values obtained during standing were averaged for the purpose of the analysis). The inhibition of standing tachycardia was used as an estimate of the degree of cardiac $\beta$-blockade. ${ }^{11}$

Venous blood samples for the determination of the serum propranolol concentration were taken at the completion of the tests, which were performed at approximately the same time of the day for each subject. All the tests were performed by the same investigator.

Tremor analysis Accelerometric signals were processed by using a Hewlett-Packard 5420 A signal analyser, ${ }^{12}$ according to a previously described procedure. ${ }^{13}$ The following parameters were determined: dominant peak frequency $(\mathrm{Hz})$ of tremor, its magnitude scaled in rms acceleration $\left(g=981 \mathrm{~cm} / \mathrm{s}^{2}\right)$ and amplitude of hand displacement $(\mathrm{cm})$. Only the data derived from the more involved hand were used for the statistical analysis of the results.

Serum propranolol assay Serum propranolol concentration was determined by high-pressure liquid chromatography according to the method described by Nygard et al. ${ }^{14}$ Statistical analysis Comparisons were always made between data obtained after equivalent periods of drug or placebo treatment (that is, the measurements obtained at the end of the 2nd and 4th week of placebo were compared with those obtained at the end of the 2 nd and 4th week of propranolol respectively). The values of amplitude and frequency of tremor were compared by using Wilcoxon test for paired differences.

Student's $t$ test for paired data was used for the calculation of changes in pulse rate. The unpaired $t$ test was used for comparing mean values of age, duration of the disorder, inhibition of standing tachycardia and serum propranolol levels between groups of patients with high and low amplitude of tremor respectively (see Results).

The relationships between amplitude and frequency of tremor, age of the patients, duration of the disorder, inhibition of standing tachycardia, serum propranolol level and response to propranolol (absolute and percentage) were evaluated by means of the Spearman's rank correlation.

\section{Results}

AMPLITUDE AND FREQUENCY OF TREMOR DURING THE PLACEBO REGIMEN

Two patients had placebo discontinued after a period of two weeks because tremor interfered seriously with their daily activities. The frequency of the dominant peak of hand tremor in the patients studied ranged from 4.2 to $10.0 \mathrm{~Hz}$ (median 7.2 $\mathrm{Hz}$ ). In any individual patient, the tremor peak frequency showed some variation on the two different occasions of recording but when all patients were considered the difference was not statistically significant. The magnitude of hand tremor at the dominant peak frequency ranged from $1 \cdot 3$ to $236 \mathrm{~g}$ $\times 10^{-3}$, which in terms of hand displacement corresponds to 0.6 to $270 \times 10^{-3} \mathrm{~cm}$ amplitude (median $4.7 \times 10^{-3} \mathrm{~cm}$ ). There was an inverse correlation between the amplitude and the frequency of the tremor $\left(r_{s}=-0.65, p<0.05\right)$ (Fig 1) whereas age of the patients and duration of the disorder did not correlate with either amplitude or frequency of tremor (data calculated on the 4 th week of placebo treatment).

In four patients the tremor amplitude during the 2nd week of placebo was found to be lower than $2 \times$ $10^{-3} \mathrm{~cm}$, that is the upper limit of physiological hand tremor in our laboratory (unpublished data). All patients had pathological values of tremor amplitude during the 4 th week of placebo treatment.

\section{THE EFFECT OF PROPRANOLOL ON TREMOR FREQUENCY AND AMPLITUDE}

Only 13 patients received propranolol at higher dosage. The drug was discontinued after two weeks of treatment in two patients because of breathlessness and for failure to attend the clinic in a further patient.

There was no significant differences between the 


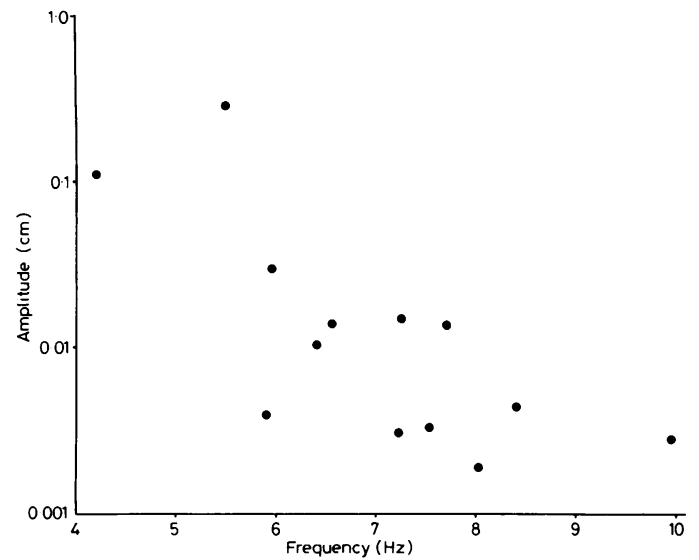

Fig 1 Relationship between amplitude (cm hand displacement) on log scale and frequency of tremor in the patients studied (data derived from measurements performed during the 4th week of placebo). $r_{s}=-0.65(N=13), p<0.05$.

dominant peak frequency of tremor observed during either dosage of propranolol and that observed during placebo. Nine out of 16 patients on propranolol $120 \mathrm{mg}$ daily and 12 out of 13 patients on propranolol $240 \mathrm{mg}$ daily had tremor amplitudes lower than those observed during the placebo regimen. When the difference in tremor amplitude was calculated as absolute change, propranolol was found to be significantly ( $p<0 \cdot 01$ ) superior to placebo only at the higher dosage regimen (240 mg daily). The median percentage changes in tremor amplitude after propranolol 120 and $240 \mathrm{mg}$ daily were $-25 \%$ (range from +55 to $-67 \%$ ) (NS) and $-45 \%$ (range from +230 to $-87 \%)(p<0.02)$ of the control (placebo) values, respectively. When the changes in tremor amplitude produced by the two dosage regimens were compared, no statistically significant difference was found.

Three patients on propranolol $120 \mathrm{mg}$ daily and one patient on propranolol $240 \mathrm{mg}$ daily had tremor amplitude virtually suppressed to less than $2 \times 10^{-3}$ $\mathrm{cm}$, that is the upper limit of physiological hand tremor in our laboratory.

Highly significant positive correlations could be found between the absolute and percentage response to propranolol $240 \mathrm{mg}$ daily and control (placebo) tremor amplitude $\left(r_{\mathrm{s}}=0.94\right.$ and 0.71 respectively, $p<0.01$ ). The relationship between absolute and percentage change in tremor amplitude and control amplitude is shown in figs 2 and 3 . The absolute and percentage responses to propranolol were also negatively correlated with the control frequency of tremor $\left(r_{s}=-0.63\right.$ and -0.53 respec-

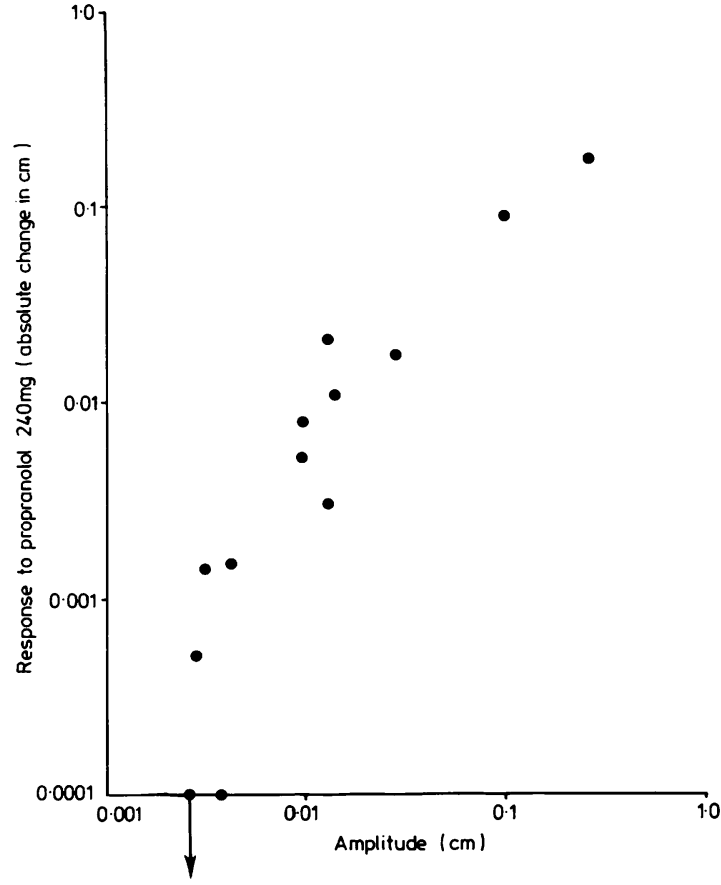

Fig 2 Relationship between absolute change in tremor amplitude (cm hand displacement as compared to the placebo value) after propranolol (240 mg daily) and control tremor amplitude $(\mathrm{cm})$ on log scales in 12 patients with essential tremor. Arrow indicates a 13th patient who showed a deterioration during. drug treatment (tremor amplitude in this subject was $26 \times 10^{-3} \mathrm{~cm}$ during placebo and $60 \times$ $10^{-3} \mathrm{~cm}$ during propranolol). $r_{s}=-0.94(N=13), p<0.01$.

tively, $\mathrm{p}<0.05$ ), but the correlation coefficients were lower than those found with amplitude. No correlation could be found between absolute or percentage response and age of the patients or duration of tremor. The table summarises the correlation between the response to propranolol, variables of essential tremor, degree of cardiac $\beta$-blockade and serum propranolol concentration.

In order to investigate further the relationship between amplitude of tremor and response to propranolol, patients were divided into two subgroups according to tremor amplitude. ${ }^{2}$ In the eight patients who had, during the 4th week of the control (placebo) period, amplitudes greater than $6 \times 10^{-3}$ $\mathrm{cm}$ hand displacement (median amplitude $13 \times 10^{-3}$ $\mathrm{cm}$, range from 9.7 to $270 \times 10^{-3} \mathrm{~cm}$; median frequency $6.4 \mathrm{~Hz}$, range $4 \cdot 2-8 \cdot 1 \mathrm{~Hz})$ propranolol $(240$ $\mathrm{mg}$ ) decreased the amplitude of the tremor of more 


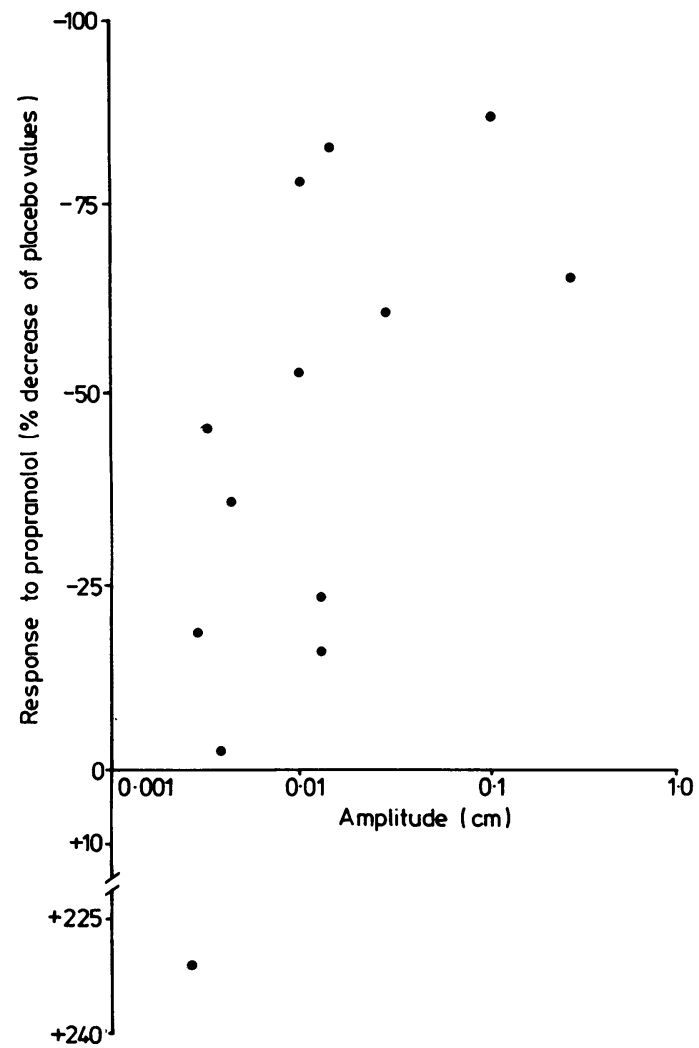

Fig 3 Relationship between percentage change in tremor amplitude (as compared to the placebo value) after propranolol (240 $\mathrm{mg}$ daily) and control tremor amplitude (cm hand displacement) on log scale in 13 patients with essential tremor.

$r_{s}=0.71, p<0.01$.

than $20 \%$ in all but one of these patients (median $65 \%$, range from -15 to $-87 \%$ ). Only two of the five patients with amplitudes below $6 \times 10^{-3} \mathrm{~cm}$ hand displacement (median amplitude $3.1 \times 10^{-3}$ $\mathrm{cm}$, range from 2.6 to $4.2 \times 10^{-3} \mathrm{~cm}$; median frequency $8.0 \mathrm{~Hz}$, range $5.9-10.0 \mathrm{~Hz}$ ) showed a decrease of tremor amplitude greater than $20 \%$. The median decrease in tremor amplitude in these patients was by $18 \%$ of the control values (range from +230 to $-45 \%$ ). There was no significant difference in age or duration of tremor between the two sub-groups.

THE EFFECT OF PROPRANOLOL ON PULSE RATE At the lower dosage (120 mg daily) the effect of propranolol on standing tachycardia was not

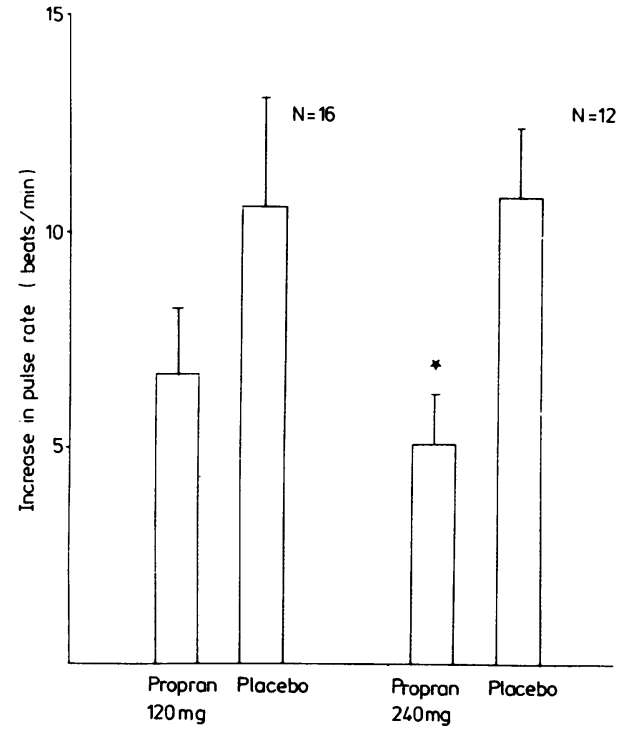

Fig 4 Increase in pulse rate on standing during propranolol (120 mg and $240 \mathrm{mg}$ ) and placebo regimens in the patients studied. Bars represent mean \pm SEM. ${ }^{*} p<0.01$ as compared to placebo.

significantly different from that of placebo. At 240 $\mathrm{mg}$ daily, propranolol significantly reduced the increase in pulse rate on standing $(p<0.01)$ (fig 4). There was no statistically significant relationship between the tremor response to either dosage of propranolol (expressed as absolute or percentage change) and the inhibition of standing tachycardia (table). The inhibition of standing tachycardia was similar in the sub-groups of patients with small and large tremor amplitude.

\section{SERUM PROPRANOLOL CONCENTRATION}

Serum levels of propranolol were found to vary from 21.0 to $164.0 \mathrm{ng} / \mathrm{ml}$ (median $44.0 \mathrm{ng} / \mathrm{ml}$ ) on daily dosage of $120 \mathrm{mg}$ and from 60.0 to $296.0 \mathrm{ng} / \mathrm{ml}$ (median $113.1 \mathrm{ng} / \mathrm{ml}$ on daily dosage of $240 \mathrm{mg}$ of the drug.

There was no statistically significant correlation between serum propranolol concentration and either the response to the drug (expressed as absolute or percentage change) or the degree of cardiac $\beta$-blockade as assessed by the inhibition of standing tachycardia (table). There was no significant difference in serum propranolol levels between the small and large amplitude sub-groups. 
Table Spearman's rank correlations between tremor response to propranolol and variables of essential tremor, inhibition of standing tachycardia and serum propranolol concentration.

\begin{tabular}{|c|c|c|}
\hline & $\begin{array}{l}\text { Response (\%) } \\
\text { to propranolol } \\
240 \mathrm{mg}(N=13)\end{array}$ & $\begin{array}{l}\text { Response (absolute) } \\
\text { to propranolol } \\
240 \mathrm{mg}(N=13)\end{array}$ \\
\hline $\begin{array}{l}\text { Amplitude } \\
\text { Frequency } \\
\text { Duration } \\
\text { Age } \\
\text { Inhibition of standing tachycardia } \\
\text { Serum propranolol concentration }\end{array}$ & $\begin{array}{l}r_{s}=0.71, p<0.01 \\
r_{s}=-0.53, p<0.05 \\
r_{s}=-0.20 \mathrm{NS} \\
r_{s}=0.25 \mathrm{NS} \\
r_{s}=-0.33 \mathrm{NS} \\
r_{s}=-0.25 \mathrm{NS}\end{array}$ & $\begin{array}{l}r_{s}=0.94, p<0.01 \\
r_{s}=-0.63, p<0.05 \\
r_{s}=0.15 \mathrm{NS} \\
r_{s}=0.22 \mathrm{NS} \\
r_{s}=-0.15 \mathrm{NS} \\
r_{s}=-0.29 \mathrm{NS}\end{array}$ \\
\hline
\end{tabular}

\section{Discussion}

In the present study, propranolol was found to produce a decrease in tremor amplitude (as compared to placebo) greater than $20 \%$ in eight out of 16 patients at a daily dosage of $120 \mathrm{mg}$ and in nine out of 13 patients at a daily dosage of $240 \mathrm{mg}$, the maximum effect being a reduction down to $13 \%$ of the control value. The dosages included in this investigation are within the range commonly employed in the treatment of essential tremor and, according to Jefferson et $a l,{ }^{4}$ they are also those at which the optimum therapeutic benefit is usually seen. Taken altogether, the results are in agreement with previous reports that only about two thirds of the patients with essential tremor respond to propranolol when the drug is given on a daily basis ${ }^{1-3}$ and that the degree of the response is variable and usually incomplete. ${ }^{15}$

The most interesting finding of our study is the demonstration that the response of essential tremor to prolonged administration of propranolol is a function of the tremor amplitude, at least in patients whose amplitude, in the absence of active treatment, exceeds $6 \times 10^{-3} \mathrm{~cm}$ hand displacement. In patients with lower amplitudes, the clinical response was unpredictable and attenuated (median decrease in tremor amplitude $17 \%$, as compared to $65 \%$ in the larger amplitude group). This differential response to propranolol could not be ascribed to pharmacokinetic factors, since serum propranolol levels and degree of cardiac $\beta$-blockade were similar in the two groups.

The tendency for the response to chronic propranolol administration to be more favourable in patients with lower frequency of tremor is likely to reflect the inverse correlation between amplitude and frequency of tremor (see Results).

These findings are in contrast with those reported by Sorensen et al, ${ }^{6}$ who showed the degree of tremor reduction after prolonged administration of propranolol to be independent of its amplitude. These authors, however, studied only five patients, a group probably too small to allow a meaningful estimate of the factors affecting the response to the drug. In contrast with previous studies, ${ }^{1278}$ we were not able to find any significant correlation between response to the drug and age of the patients or duration of the disorder. The fact that in our study the response to propranolol did not correlate with the serum concentration of the drug is in agreement with the results reported by others. ${ }^{46}$

All patients but one had previously taken part in a single-dose study on the effect of propranolol in essential tremor.' Since the same methods of assessment were used on that occasion, a comparison between the results obtained in the two studies is of considerable interest. In agreement with the data obtained in the chronic situation, the response observed after a single dose of propranolol was better in the patients with larger tremor amplitude. On the whole, the efficacy of the drug was greater after acute than after chronic administration. It is particularly noteworthy that after acute administration propranolol was clearly superior to placebo in reducing essential tremor at a dose of $120 \mathrm{mg}$, whereas during prolonged treatment the effect was evident only at a dosage of $240 \mathrm{mg}$ daily. It is reasonable to assume that this discrepancy may be due mainly to differences in the experimental design (in the acute study propranolol was given as a single dose and not as three divided doses), resulting in different serum levels of the drug. Indeed, serum concentrations of propranolol in the range of that obtained following a single oral dose $(120 \mathrm{mg})^{9}$ were achieved with 240 $\mathrm{mg}$ of the drug when given chronically. Nevertheless a higher prevalence of the "placebo effect" after prolonged administration of the drug could partially account for this differential response.

On the basis of the response to acute and chronic propranolol administration it may be postulated that there are at least two subtypes of essential tremor. The first one, characterized by smaller amplitude and usually (but not necessarily) higher peak frequency, clinically resembles "exaggerated" physiological tremor and may be considered as a "primary" enhancement of the latter. This subtype differs from "secondarily" exaggerated physiologi- 
cal tremor (for example, the tremor seen after catecholamines infusion, stress etc) in that it does not respond to acute and chronic propranolol. The second subtype of essential tremor, showing larger amplitude and usually (but not necessarily) lower peak frequency, responds to adequate doses of oral propranolol to an extent which is directly proportional to its amplitude. Whether the two subtypes are separate pathological entities or a continuum, the more severe form simply representing the progression of the same disorder, is unknown and deserves further investigation. The lack of reponsiveness of one subtype to propranolol may underline the existence of separate pathophysiological mechanisms or simply reflect the inability of the drug to affect any type of tremor when the amplitude is below a given "cut-off" value.

One important question from the practical point of view is whether patients with small amplitude of essential tremor should be treated with propranolol. Our findings clearly indicate that such tremor does not respond to the drug. It should be pointed out, however, that in many of these patients the tremor can be aggravated and become severely disabling under conditions associated with adrenergic stimulation (for example, anxiety and stress). Since this "secondary" exaggeration is known to respond to $\beta$-blockers, ${ }^{16}$ the choice whether to treat or not to treat should depend on careful evaluation of the medical history, with particular regard to the identification of precipitating factors.

We thank the physicians at the National Hospital, Queen Square, London, for allowing us to study patients under their care. We also thank the staff of the pharmacy at the National Hospital for their assistance and Dr $\mathbf{J}$ Ward, Department of Clinical Pharmacology, The Groby Road Hospital, Leicester, for carrying out the propranolol assays. Dr S Calzetti has been supported by a European Science Foundation fellowship, European Training Programme in Brain and Behaviour Research 19801981 (Strasbourg, France).

\section{References}

${ }^{1}$ DuPont E, Hansen HJ, Dalby MA. Treatment of benign essential tremor with propranolol. Acta Neurol Scand 1973;49:75-84.
${ }^{2}$ Teravainen H, Fogelholm R, Larsen A. Effect of propranolol on essential tremor. Neurology (Minneap) 1976;26:27-30.

${ }^{3}$ Winkler GF, Young RR. Efficacy of chronic propranolol therapy in action tremors of familial, senile or essential varieties. $N$ Engl J Med 1974;290:984-88.

${ }^{4}$ Jefferson D, Jenner P, Marsden CD. Relationship between plasma propranolol concentration and relief of essential tremor. J Neurol Neurosurg Psychiatry 1979;42:831-7.

${ }^{5}$ McAllister RG Jr, Markesbert WR, Ware RW, Howell RM. Suppression of essential tremor by propranolol: correlation of effect with drug plasma levels and intensity of beta-adrenergic blockade. Ann Neurol 1977;1:160-6.

${ }^{6}$ Sorensen PS, Paulson OB, Steiness E, Jansen EC. Essential tremor treated with propranolol: lack of correlation between clinical effect and plasma propranolol levels. Ann Neurol 1981;9:53-57.

${ }^{7}$ Murray TJ. Long-term therapy of essential tremor with propranolol. Can Med Assoc J 1976;115:892-4.

${ }^{8}$ Larsen A. Beta-blockers, tremor and neuromuscular transmission. A clinical and experimental study. Academic Dissertation, University of Helsinki, Helsinki, 1980.

${ }^{9}$ Calzetti S, Findley LJ, Gresty MA, Perucca E, Richens A. The effect of a single oral dose of propranolol on essential tremor. A double-blind controlled study. Ann Neurol 1982 (In press).

${ }^{10}$ Calzetti S, Findley LJ, Perucca E, Richens A. Controlled study of metoprolol and propranolol following prolonged administration in patients with essential tremor. J Neurol Neurosurg Psychiatry 1982 (In press).

" Carruthers SG, Ghosal AG, McDevitt DG, Nelson JK, Shanks RG. The assessment of beta-adrenoceptor blocking drugs in hyperthyroidism. Br J Clin Pharm 1974;1:93-98.

${ }^{12}$ Hewlett-Packard. Digital signal analyser: user's guide. HP 05420-900 July, 1978.

${ }^{13}$ Calzetti S, Findley LJ, Gresty MA, Perucca E, Richens A. Metoprolol and propranolol in essential tremor: a double-blind, controlled study. J Neurol Neurosurg Psychiatry 1981;44:814-19.

${ }^{14}$ Nygard G, Shelver WM, Wahba Khalil SK. Sensitive high pressure liquid-chromatographic determination of propranolol in plasma. J Pharm Sci 1979;68:379 81.

${ }^{15}$ Murray TJ. Essential tremor. In Barbeau A (Ed): Disorders of Movement Lancaster, MTP Press, 1981;151-70.

${ }^{16}$ Marsden CD, Gimlette TMD, McAllister RG, Owen DAL, Miller TN. Effect of $\beta$-adrenergic blockade on finger tremor and achilles reflex time in anxious and thyrotoxic patients. Acta Endocr (Copenh) 1968;57:353-62. 